\title{
Erectile dysfunction in atrial fibrillation: A risk factor for stroke or a reflection of stroke risk factors?
}

\author{
Richard A. Brown, Gregory Y.H. Lip \\ University of Birmingham Center for Cardiovascular Sciences, \\ City Hospital, Birmingham, United Kingdom
}

Article p. 446

Atrial fibrillation (AF) confers a 5-fold increase in the risk of stroke and thromboembolism and a 2 -fold increase in mortality due mainly to stroke and heart failure. Oral anticoagulation with well managed dose adjusted vitamin $\mathrm{K}$ antagonist or a non-vitamin $\mathrm{K}$ antagonist highly effective in reducing this risk. Unfortunately, despite oral thromboprophylaxis, adverse cardiovascular (CV) events in AF patients is approximately $4 \%$ [1].

In the current issue of "Cardiology Journal", Szymański et al. [2] report a higher prevalence of erectile dysfunction (ED) among older patients with increasing thromboembolic risk. In its present form the paper does little more than highlight the association between ED and older age since this is the only component of either the $\mathrm{CHADS}_{2}$ or $\mathrm{CHA}_{2} \mathrm{SD}_{2}$-VASc scores that is significantly different between older patients (defined as $\geq 65$ by the authors) with and without ED and therefore driving the outcome in this population. In the younger group (defined as $<65$ by the authors), the age difference is not significant enough to create a difference in the overall $\mathrm{CHADS}_{2}$ or $\mathrm{CHA}_{2} \mathrm{SD}_{2}$-VASc risk score between patients with and without ED. Also notable is the absence of a multivariate analysis to adjust for the difference in waist circumference since several measures of adiposity are negatively correlated with endothelial function and waist circumference reduction has been shown to be associated with improved endothelial function in certain groups [3]. Moreover, increased adiposity creates a procoagulant state and is linked to over expression of proinflammatory markers of endothelial dysfunction such as fibrinogen, $\mathrm{C}$ reactive protein and von Willebrand Factor (vWF), possibly via a mechanism involving the CD40 ligand $[4,5]$. Furthermore, whilst it may be appropriate to consider ED as an additional marker of a prothrombotic state, it is difficult to see how a diagnosis of ED would influence the clinical decision-making process in patients over 65 with $\mathrm{AF}$ (other than to prompt treatment of the ED itself), who would already qualify for oral anticoagulation according to international guidelines. Also, it would seem counterintuitive that ED would add much predictive power to the $\mathrm{CHA}_{2} \mathrm{SD}_{2}$-VASc score given that women are at a higher overall risk of thromboembolic stroke than men for reasons which are unclear but may be related to hormone therapy and sex differences in hemodynamics and $\mathrm{CV}$ remodeling [6]. ED, like stroke, is an outcome of cumulative vascular risk factors including $\mathrm{AF}$.

The $\mathrm{CHADS}_{2}$ and the $\mathrm{CHA}_{2} \mathrm{SD}_{2}$-VASc scores have been shown to predict to thromboembolic risk in patients with $\mathrm{AF}$, with the $\mathrm{CHA}_{2} \mathrm{SD}_{2}$-VASc score improving risk stratification at the 'low risk' end of the spectrum [7]. In addition, Roldán et al. [8] have previously reported a significant positive correlation between vWF and the $\mathrm{CHADS}_{2}$ score supporting the association between increasing thromboembolic risk and vascular dysfunction.

The inherent threat to vascular health associated with the presence of these risk factors is common to all vascular territories so it is perhaps not surprising that accumulation of thromboembolic risk factors is associated with $\mathrm{ED}$ - itself, an independent marker of endothelial dysfunction that

Address for correspondence: Prof. Gregory Y.H. Lip, University of Birmingham Center for Cardiovascular Sciences, City Hospital, Birmingham, United Kingdom, tel: +44 121507 5080, fax: +44 121554 4083, e-mail: g.y.h.lip@bham.ac.uk Received: 05.07.2015 Accepted: 06.07.2015 
is well recognised in AF [9]. Despite the analytical shortcomings the paper raises an interesting mechanistic hypothesis regarding occlusion of the penile arteries by microthrombi according to the artery size hypothesis. This is certainly plausible in terms of Virchow's triad of thrombogenesis since AF leads directly to blood stasis and hypercoagulability within the left atrium (LA). Moreover, evidence from Lim et al. [10] has demonstrated that thrombin generation occurs in humans to a greater extent in the LA in response to $\mathrm{AF}$ thus supporting the potential for microthrombi embolisation.

Over the last decade, it has become increasingly apparent that AF is also associated with systemic inflammation and endothelial dysfunction irrespective of traditional CV risk factors. For example, permanent $\mathrm{AF}$ has been shown to be an independent predictor of impaired brachial artery flow mediated dilatation [11] whilst plasma levels of soluble E-selectin and $\mathrm{vWF}$ are raised in all forms of AF [12]. Furthermore, in porcine models, rapid atrial tachycardia (simulated by pacing) has been shown to enhance the local release of vasoactive substances such as angiotensin II [13], whilst also down-regulating the expression of nitric oxide synthase (NOS) expression and subsequent nitric oxide production [14]. This not only creates oxidative stress leading to subsequent left ventricular microvascular flow abnormalities but also leads to loss of the antithrombotic properties of NOS, thus contributing to hypercoagulability. In humans, whilst rapid atrial rates increase platelet activation (particularly in the LA), the additive effects of endothelial dysfunction and inflammation seem to occur only in the presence of AF [10]. Conversely, both macrovascular and microvascular indices of endothelial function have been found to predict atrial high rate episodes in patients with dual chamber pacemakers [15]. It is therefore likely that a self-perpetuating phenomenon exists (as with many scenarios in medicine) whereby continued AF leads to declining endothelial function which further contributes to continuation of AF. Importantly, improved endothelial function has been demonstrated after restoration of sinus rhythm in AF patients with some studies showing an increase in circulating endothelial progenitor cells post cardioversion suggesting a role for these reparative cells [16].

Inflammation is an important potential link between $\mathrm{AF}$ and impaired endothelial function since activated inflammatory cells and mediators such as tumor necrosis factor $\alpha$, interleukin (IL)-2, IL-6, IL-8, and/or monocyte chemoattractant protein (MCP)-1, that develop during arrhythmia promote endothelial injury and confer a prothrombotic state [1].

Recently, there has been considerable progress in identifying genetic variants of $\mathrm{CV}$ disease. Micro RNAs (miRNA) are endogenously produced small molecules that specifically bind to miRNA and have assumed a fundamental role in the gene expression regulatory network [1]. They have been linked to atherogenesis, induction and perpetuation of $\mathrm{AF}$, and, more recently, described as prognostic markers of adverse $\mathrm{CV}$ events in anticoagulated $\mathrm{AF}$ patients [1].

Whether or not $\mathrm{ED}$ is predictor of stroke in $\mathrm{AF}$ patients remains to be seen. There are conflicting studies in non AF patients. The pathophysiology by which this common arrhythmia fulfils the components of Virchow's triad is multi-faceted and due, in part, to the complex structural and electrical atrial remodeling that occurs in this condition. Micro RNA may represent a therapeutic target for reducing thromboembolic risk in the future but at the moment it is early days.

\section{Conflict of interest: None declared}

\section{References}

1. Roldán V, Arroyo AB, Salloum-Asfar S et al. Prognostic role of MIR146A polymorphisms for cardiovascular events in atrial fibrillation. Thromb Haemost, 2014; 112: 781-788.

2. Szymański FM, Filipiak KJ, Platek AE, Kotkowski M, Opolski G. Can thromboembolic risk be associated with erectile dysfunction in atrial fibrillation patients? Cardiol J, 2015; 22: 446-452.

3. Miyazaki S, Hiasa Y, Takahashi T et al. Waist circumference reduction is more strongly correlated with the improvement in endothelial function after acute coronary syndrome than body mass index reduction. J Cardiol, 2010; 55: 266-273.

4. Morange PE, Alessi MC. Thrombosis in central obesity and metabolic syndrome: mechanisms and epidemiology. Thromb Haemost, 2013; 110: 669-680.

5. Missiou A, Wolf D, Platzer I et al. CD40L induces inflammation and adipogenesis in adipose cells: A potential link between metabolic and cardiovascular disease. Thromb Haemost, 2010; 103: 788-796.

6. Cove CL, Albert CM, Andreotti F, Badimon L, Van Gelder IC, Hylek EM. Female sex as an independent risk factor for stroke in atrial fibrillation: Possible mechanisms. Thromb Haemost, 2014; 111: 385-391.

7. Olesen JB, Torp-Pedersen C, Hansen ML, Lip GY. The value of the CHA2DS2-VASc score for refining stroke risk stratification in patients with atrial fibrillation with a CHADS2 score 0-1: A nationwide cohort study. Thromb Haemost, 2012; 107: 1172-1179.

8. Roldan V, Marin F, Garcia-Herola A, Lip GY. Correlation of plasma von Willebrand factor levels, an index of endothelial damage/dysfunction, with two point-based stroke risk stratification scores in atrial fibrillation. Thromb Res, 2005; 116: 321-325. 
9. Lin W-Y, Lin C-S, Lin C-L, Cheng S-M, Lin W-S, Kao C-H. Atrial fibrillation is associated with increased risk of erectile dysfunction: A nationwide population-based cohort study. Int J Cardiol, 2015; 190: 106-110.

10. Lim HS, Willoughby SR, Schultz C et al. Effect of atrial fibrillation on atrial thrombogenesis in humans: Impact of rate and rhythm. J Am Coll Cardiol, 2013; 61: 852-860.

11. Freestone B, Chong AY, Nuttall S, Lip GYH. Impaired flow mediated dilatation as evidence of endothelial dysfunction in chronic atrial fibrillation: Relationship to plasma von Willebrand factor and soluble E-selectin levels. Thromb Res, 2008; 122: 85-90.

12. Freestone B, Chong AY, Nuttall S, Blann AD, Lip GY. Soluble E-selectin, von Willebrand factor, soluble thrombomodulin, and total body nitrate/nitrite product as indices of endothelial damage/dysfunction in paroxysmal, persistent, and permanent atrial fibrillation. Chest, 2007; 132: 1253-1258.
13. Goette A, Bukowska A, Dobrev D et al. Acute atrial tachyarrhythmia induces angiotensin II type 1 receptor-mediated oxidative stress and microvascular flow abnormalities in the ventricles. Eur Heart J, 2009; 30: 1411-1420.

14. Cai H, Li Z, Goette A et al. Downregulation of endocardial nitric oxide synthase expression and nitric oxide production in atrial fibrillation: Potential mechanisms for atrial thrombosis and stroke. Circulation, 2002; 106: 2854-2858.

15. Krishnamoorthy S, Khoo CW, Lim HS, Lip GY. Predictive value of atrial high-rate episodes for arterial stiffness and endothelial dysfunction in dual-chamber pacemaker patients. Eur J Clin Invest, 2014; 44: 13-21.

16. Watson T, Shantsila E, Blann A, Lip GY. Circulating progenitor cells in patients with atrial fibrillation and their relation with serum markers of inflammation and angiogenesis. Thromb Haemost, 2010; 104: 327-334. 